of congruent or symmetric divisions begin with $1,1,2,3,6,9$, $24, \cdots$ The divisions themselves may be classified, for a fixed point, according to the next point in the same subset. We obtain once more the recursion formula leading to $c_{n}=C_{2 n, n} /(n+1)$.

UNIVERSITY OF JERUSALEM

\title{
INDEPENDENCE OF RESULTANTS
}

TH. MOTZKIN

In this note it is proved that the resultants of $m$ forms, of a sufficiently high degree, in $n$ variables are independent functions of the coefficients of the forms. The proof demands some lemmas on irreducible manifolds, and on monomial manifolds. A monomial manifold is defined by equalities between monomials, that is, products of powers of the coordinates.

In the evaluation of the number of independent hypersurface cross ratios and generalized intersections given in two other notes ${ }^{1} I$ have assumed the above theorem to be true for $2 n-1 \leqq m \leqq 2 n+1$ and, in the case of intersections, where one of the forms is supposed to be linear for $2 n-2 \leqq m \leqq 2 n$.

The resultant $r=r\left(a_{1}, \cdots, a_{n}\right)$ of $n$ forms $a_{k}$ of positive degree $d_{k}$ in $n$ variables $x_{k}$ within an (algebraically) closed field is uniquely defined as an irreducible polynomial in the coefficients of the forms such that $r=1$ if $a_{k}$ is a power of $x_{k}$ and $r=0$ if and only if values $x_{k}$, not all of them 0 , exist for which all $a_{k}=0$. The resultant is almost symmetric, that is, it becomes $r$ or $-r$ if the forms are permuted. The resultant is multiplicative in the sense that if a form $a_{k}$ is a product of forms, then $r$ is the product of the resultants obtained by replacing $a_{k}$ by each of its factors. ${ }^{2}$

THEOREM 1. The $C_{m, n}$ resultants that can be formed of $m$ forms $a_{1}, \cdots, a_{m}$ in $n$ variables are independent functions of the coefficients of the forms, provided that the degree $d_{k}$ of $a_{k}$ exceeds a bound depending only on $k$ and $n$.

Received by the editors September 16, 1946.

${ }^{1}$ The hypersurface cross ratio, Bull. Amer. Math. Soc. vol. 51 (1945) pp. 976-984, \$3.6, and Relations between hypersurface cross ratios, and a combinatorial formula for partitions of a polygon, for permanent preponderance, and for non-associative products, Bull. Amer. Math. Soc. vol. 54 (1948) pp. 352-360.

${ }^{2}$ All these properties of the resultant are well known. Cf. also $\$ 1.1$ of the before mentioned note The hypersurface cross ratio. 
We shall prove the theorem for $d_{k} \geqq C_{k-1, n-1}$. Thus $d_{1}, \cdots, d_{n}$ are arbitrary.

We have to show that there is no algebraic relation fulfilled by every system of $C_{m, n}$ values attained by the resultants of $m$ forms of the given degrees. We say more shortly that almost every system is attained: generally, a subset $P$ of a set $Q$ will be said to include almost every element of $Q$ if every algebraic relation fulfilled by all elements of $P$ is also fulfilled by all elements of $Q .{ }^{3}$

As forms, we choose products of linear forms, the first $n$ of which shall be powers. That for such products almost every system of values of the resultants is attained is trivial if $m=n$ or if $n=1$ and will be supposed true for $m-1$ if $m>n>1$.

Choose, accordingly, $a_{1}, \cdots, a_{m-1}$ so that the $b=C_{m-1, n}$ resultants formed by these products of linear forms have the desired values; these values $q_{1}, \cdots, q_{b}$ are restricted by the possibility of the choice and are, furthermore, required to be different from 0 . Still $q_{1}, \cdots, q_{b}$ can be almost every system. There remain $c=C_{m-1, n-1}>1$ resultants $r_{1}, \cdots, r_{c}$ involving $a_{m}$; they are the coordinates of a point $r$ in the affine space $S$ of $c$ dimensions. We show that:

(1) The points $r$ corresponding to all $a_{m}$ of degree one, and to the chosen $a_{1}, \cdots, a_{m-1}$, form a set ( $r$ ) "defining" (see below) an irreducible algebraic manifold $R$ that contains, for every $k=1, \cdots, c$, a point with $r_{k}=0$ and no other zero coordinate.

(2) For every such set $(r)$, almost every point of $S$ is a product of $c$ points (or any given number greater than $c$ of points) of $(r)$.

Here the product of two points $r_{1}, \cdots, r_{c}$ and $s_{1}, \cdots, s_{c}$ is the point $r_{1} s_{1}, \cdots, r_{c} s_{c}$. From (1) and (2) follows by the multiplicative property of the resultant the existence, for any given $d_{m} \geqq c$, and for almost every system of numbers $r_{1}, \cdots, r_{c}$, of a product $a_{m}$ of $d_{m}$ linear forms for which the values of the resultants are $r_{1}, \cdots, r_{c}$. Now if there should hold a relation between the values $q_{1}, \cdots, q_{b}$, $r_{1}, \cdots, r_{c}$ attained, then for every $q_{1}, \cdots, q_{b}$ there would be a relation between $r_{1}, \cdots, r_{c}$, except for those $q_{1}, \cdots, q_{b}$ for which the latter relation becomes $0=0$; but that would give one or more relations between $q_{1}, \cdots, q_{b}$.

${ }^{3}$ Not, as usual in algebraic geometry, if the complementary set $Q-P$ has less dimensions than $Q$, which, for an irreducible manifold $Q$, is a stronger condition (presumably also fulfilled in our case). By the present definition $\boldsymbol{P}$ may even, for irreducible $Q$ and if points with coordinates outside the given field are admitted, be a single point (a "general point" of $Q$ ). Compare the three further definitions of "almost every": except a set of zero measure, or density, or except a finite set.

Not every system of values need be attained by a system of independent polynomials, for example $(x, x y)$ is never $(0,1)$. 
To prove (1) we remark that since no resultant of $n$ forms from among $a_{1}, \cdots, a_{m-1}$ vanishes, no point $x_{1}, \cdots, x_{n}$ of the projective $(n-1)$-space annihilates more than $n-1$ of these forms. Hence $n-1$ forms cannot vanish for all the points of a straight line; therefore, being products of linear forms, they vanish for the points of a finite set $S_{k}$. Clearly no two sets $S_{j}$ and $S_{k}$ have a point in common. Hence there exists, for any $S_{k}$, a hyperplane $a_{m}=0$ through one of its points that does not pass through any point of the other sets, which is equivalent to saying that $r_{k}=0$ for the given $k$ and only for that $k$.

The irreducibility of the algebraic manifold $R$ defined by the set $(r)$ follows from (3), taking $Q$ as the affine $n$-space itself. ${ }^{4}$

The smallest algebraic manifold $R$ containing all points of a given set $(r)$ is called the manifold defined by $(r)$; obviously, almost every point of $R$ belongs to $(r) .{ }^{5}$ In particular, if $(r)$ consists of all the points whose coordinates are the values of $c$ given polynomials $f_{1}, \ldots, f_{c}$ in the coordinates $b_{1}, \cdots, b_{n}$ of a point of a given manifold $Q$ of the affine $n$-space, and if a set $(q)$ contains almost every point of $Q$ then, to the points of $(q)$, there still corresponds a set containing almost every point of $R$. For any additional relation for the points of $R$ can be read as a relation restricting the points of $Q$.

(3) If $Q$ is irreducible, then $R$ is irreducible too. ${ }^{6}$

For assume a polynomial $F\left(r_{1}, \cdots, r_{c}\right)$ to vanish for every point of $R$. Then $G=F\left(f_{1}, \cdots, f_{c}\right)=0$ for every point of $Q$. If $F$ is a product $F_{1} F_{2}$, then also $G=G_{1} G_{2}$, and either $G_{1}$ or $G_{2}$, say $G_{1}$, vanishes for every point of $Q$. Hence $F_{1}=0$ for every point of $(r)$ and therefore also for every point of $R$.

${ }^{4}$ It can even be shown that $(r)$ and $R$ are identical. Indeed, every resultant $r_{k}$ is a form $f_{k}$ of positive degree $g_{k}$ in the coefficients $b_{1}, \cdots, b_{n}$ of the linear form $a_{m}$. Instead of $f_{k}=r_{k}$ we write homogeneously $r_{0} f_{k}=r_{k} b_{0}^{g_{k}}$. This is an algebraic correspondence between the whole projective $n$-space and an algebraic manifold of the projective $c$-space, the equations of which are obtained by eliminating $b_{0}, \cdots, b_{n}$ from the equations of the correspondence. This manifold consists of the $(c-1)$-dimensional part $r_{0}=0$, corresponding to all systems with $b_{0}=0$, and of a manifold $R$. Now all $f_{k}=0$ only if $b_{1}=\cdots=b_{n}=0$; otherwise there would exist a hyperplane through one point at least of each set $S_{k}$, whereas the $n$ sets $S_{k}$ defined by any $n-1$ from among the forms $a_{1}, \cdots, a_{n}$, which are powers of linear forms, are single points not on a common hyperplane. Hence to every point of $R$ with $r_{0} \neq 0$ there corresponds a system $b_{0}, \cdots, b_{n}$ with $b_{0} \neq 0$; that is, the nonhomogeneous coordinates of the point are values attained by the resultants belonging to a linear form $a_{m}$.

${ }^{5}$ By two theorems of Hilbert, every algebraic manifold in a space over a closed field may be given either by a finite system of equations or by all its points with coordinates within the field.

6 The example $f_{1}=b_{1}, f_{2}=b_{1} b_{2}$ shows again that not every point of $R$ is necessarily a point of $(r)$. Other examples are given in footnote 7 . 
We shall use the symbol $(Q, R)$ for the set of all points $\left(q_{1}, \cdots, q_{b}\right.$, $\left.r_{1}, \cdots, r_{c}\right)$ with $\left(q_{1}, \cdots, q_{b}\right)$ in a given set $Q$ of $b$-space and $\left(r_{1}, \cdots, r_{c}\right)$ in a given set $R$ of $c$-space. Evidently, if $Q$ and $R$ are algebraic manifolds, then $(Q, R)$ is also an algebraic manifold.

If $Q$ is defined by $(q)$ and $R$ by $(r)$, then $((q),(r))$ defines $(Q, R)$. For if $F(q, r)=0$ for every point of $(q)$ and every point of $(r)$, and if $q$ is a point of $(q)$ (here we admit only points with coordinates in the given field), then $F(q, r)=0$ may be considered as an equation for $r$; since it holds for every point of $(r)$, it holds also for every point of $R$. Now if $r$ is a point of $R$, then $F(q, r)=0$ for every point of $(q)$, hence also for every point of $Q$.

(4) If $Q$ and $R$ are irreducible, then $(Q, R)$ is also irreducible.

For let $F(q, r)=0$ for all points of $(Q, R)$. For every $q$ in $Q$ this is an equation for $r$; all these equations together are fulfilled by the points of an algebraic manifold $E$ of $c$-space, which contains $R$. If $F=F_{1} F_{2}$, then every point of $E$ must, because of the irreducibility of $Q$, belong to at least one of $E_{1}$ and $E_{2}$ (defined similarly). Since $R$ is irreducible and contained in $E, R$ is contained in at least one of $E_{1}$ and $E_{2}$, Q.E.D.

The product $Q R$ of two algebraic manifolds $Q$ and $R$ of the same affine space is the manifold "defined" by the points $q r$ with $q$ in $Q$ and $r$ in $R .{ }^{7}$ Now $Q R$ is obtained from $(Q, R)$ by the formation of products, that is, of special polynomials: hence by (4) and (3) we see that:

If $Q$ and $R$ are irreducible, then $Q R$ is irreducible, ${ }^{8}$

and that $Q R$ can be defined already by almost every point of $Q$ and of $R$. In particular, if $Q$ is a product, $Q R$ can be defined by those points of $Q$ which are products of points of the factors of $Q$. Hence the multiplication of manifolds is associative.

The assertion (2) can now be written $R^{c}=S$. To see that this implies $R^{c+1}=\cdots=S$ note that already $R^{c} r=S r=S$ provided that $R$ contains a point $r$ with no vanishing coordinate. But if it did not $R$

7 The product $Q R$ may contain exceptional points not of the form $q r$. The point $x=y=1$ is, for example, exceptional for the line $y=x+1$ as $Q$ and the hyperbola $x+y=x y$ as $R$, or for $x y+2=4 x+2 y$ as $Q=R$; in both cases $Q R$ is the whole plane. If, for two points of $Q$ and $R$ with homogeneous coordinates $q_{0}, \cdots, q_{c}$ and $r_{0}, \cdots, r_{c}$, there is never $q_{0} r_{0}=\cdots=q_{c} r_{c}=0$, then there are no exceptional points, and the points $p$ of $Q R$ are given by $p_{0}: \cdots: p_{c}=q_{0} r_{0}: \cdots: q_{c} r_{c} ;$ otherwise, however, the latter equations are fulfilled by every point of the space. An example for this case, but without exceptional points, is $x y=1$ as $Q=R=Q R$ ( $=\bar{R}$, as defined in the sequel).

8 If the field of the coefficients of the equations is not closed and if points with coordinates outside the field are admitted, then $(Q, R)$ and $Q R$ may be reducible. An example is the field of rational numbers with $x^{2}=2$ as $Q=R$ and $x= \pm 2$ as $Q R$. 
would be decomposable into its (nonvoid) intersections with $r_{1}$ $=0, \cdots, r_{c}=0 .{ }^{9}$

It therefore remains to be proved that $R^{c}=S$. Neither this conclusion nor the suppositions of (2) specified in (1) are affected if $R$ is replaced by $R / r$, where $r$ is a point of $R$ with no zero coordinate. Hence we may suppose that $R$ contains the point 1 all of whose coordinates are 1.

A monomial relation is a relation $r_{1}^{h_{1}} . \cdots \cdot r_{c}^{h_{c}}=1$ with integral exponents. It may be written as an equation with non-negative exponents. If the coordinates of the points $q$ and $r$ satisfy the equation, the same is true for $q r$.

If $r_{k}$ is a factor of one of its sides, then the equation is fulfilled by no point with $r_{k}=0$ and all other coordinates different from 0 . Hence the only monomial relation satisfied by all points of the manifold $R$ of $(7)$ is the identical relation $1=1\left(h_{1}=\cdots=h_{c}=0\right)$. We may express this by saying that the monomial closure $\bar{R}$ of $R$ is $S$, where the monomial closure of a set is the manifold defined by the monomial relations fulfilled by all points of the set.

The assertion (2) is therefore, in virtue of (5), a consequence of:

THEOREM 2. If an irreducible manifold $R$ of $c$-space contains 1 , then $R^{c}=\bar{R} \cdot{ }^{10}$

The theorem follows by remarking that $\bar{R}$ contains every power $R^{d}$ and that $R^{d+1}$ contains $R^{d}$ and has more dimensions than $R^{d}$ as long as $R^{d} \neq \bar{R}$. To see this it will be enough to show that $R^{2} \neq R$ if $R \neq \bar{R}$, whence it follows that $\left(R^{d}\right)^{2} \neq R^{d}$ if $R^{d} \neq \bar{R}$. For if the irreducible manifold $R^{d+1}$, which contains $R^{d}$, had the same number of dimensions, it would follow that $R^{d}=R^{d+1}=R^{d+2}=\cdots=R^{2 d}$.

But if $R^{2}=R$ and $R$ is irreducible and contains 1 , then obviously $R=R^{0}$, where $R^{0}$ is the multiplier manifold defined by the points $r$ with $R r=R$. Since we shall presently see that $R^{0}=\overline{R^{0}}$, we have $R=\bar{R} .{ }^{11}$

${ }^{9}$ For a manifold $R$ with no such point $r$, no power equals $S$, since every power is contained in $r_{1} \cdots \cdot r_{c}=0$.

${ }^{10}$ Since every product of points of $\bar{R}$ fulfils the monomial relations defining $\bar{R}$, we have $\bar{R}^{2}=\bar{R}, R^{c+1}=R^{c+2}=\cdots=\bar{R}$.

For a manifold $R r$ "proportional" to $R$, the powers $(R r)^{d}=\bar{R} r^{d}, d \geqq c$, are disjoint and either periodic (if a power of $r$ is a point of $\bar{R}$ ) or all different. In the latter case they do not exhaust $\overline{R r}$, which must also contain $\bar{R} r^{d}$ (possibly multiplied by roots of unity) for fractional $d$.

${ }^{11}$ More generally, the product $Q R$ of two irreducible manifolds has more dimensions than $R$, except if $Q$ is either proportional to a submanifold of $R^{0}$ or contained in $q_{0} \cdots \cdots q_{c}=0$. For if $q$ and $q^{\prime}$ are points of $Q$ without zero coordinates, then $Q R$ has certainly more dimensions than $R$ unless $q R=q^{\prime} R$, or $R\left(q / q^{\prime}\right)=R$. 
Finally, why, for every algebraic manifold $R$, is there always $R^{0}=\overline{R^{0}}$ ? Let $R$ be given by a system of equations of the form $\sum c_{\nu} \rho_{\nu}$ $=0$, where the $\rho_{v}$ are monomials in $r_{1}, \cdots, r_{c}$ with non-negative exponents. The product $R s$ of $R$ and a point $s_{1}, \cdots, s_{c}$ with no zero coordinate is then given by the corresponding equations $\sum c_{\nu} \rho_{\nu} \sigma_{\nu}=0$, where $\sigma_{\nu}$ is formed from $s_{1}, \cdots, s_{c}$ in the same way as $\rho_{\nu}$ from $r_{1}, \cdots, r_{c}$. The equations may be written $\sum d_{\mu} \sigma_{\mu}=0$, where all $\sigma_{\mu}$, for the given $s$, have different values. If $R s=R$, then the points of $R$ fulfil the equations $\sum d_{\mu}=0, \sum d_{\mu} \sigma_{\mu}=0, \sum d_{\mu} \sigma_{\mu}^{2}=0, \cdots$. Since the Vandermonde determinant of the $\sigma_{\nu}$ is different from 0 , this implies that every $d_{\mu}$ separately equals 0 . If $R$ was given from the outset by shortest equations, then the $\sigma_{\mu}$ belonging to the same equation must be equal; and the equalities between them form a system of monomial relations whose solutions without a vanishing coordinate are the points $s$ with $R s=R$. If $R s=R$ for an $s$ with some zero coordinates, then the corresponding coordinates of every point of $R$ vanish and $R^{0}$ is given by monomial relations between the other coordinates. Being given by monomial relations, the multiplier manifold $R^{0}$ is a monomial manifold and equals its own monomial closure.

UNIVERSITY OF JERUSALEM 